REGARDS

SUR LECONOMIE ALLEMANDE

BULLETIN ECONOMIQUE DU CRAC

\section{Regards sur l'économie allemande}

Bulletin économique du CIRAC

$83 \mid 2007$

Varia

\title{
Les PME allemandes : acteurs de la mondialisation
}

Isabelle Bourgeois et René Lasserre

\section{OpenEdition}

Journals

Édition électronique

URL : http://journals.openedition.org/rea/585

DOI : $10.4000 /$ rea. 585

ISBN : 978-2-8218-0862-1

ISSN : 1965-0787

Éditeur

CIRAC

Édition imprimée

Date de publication : 1 octobre 2007

Pagination : 11-25

ISSN : 1156-8992

Référence électronique

Isabelle Bourgeois et René Lasserre, «Les PME allemandes : acteurs de la mondialisation », Regards sur l'économie allemande [En ligne], 83 | octobre 2007, document 2, mis en ligne le 01 octobre 2009, consulté le 19 avril 2019. URL : http://journals.openedition.org/rea/585 ; DOI : 10.4000/rea.585 


\title{
Les PME allemandes : acteurs de la mondialisation
}

\author{
Isabelle Bourgeois, René Lasserre
}

\begin{abstract}
L'Allemagne doit son titre de championne du monde à l'export à ses innombrables entreprises du Mittelstand au nom souvent peu connu du grand public. Certes, dans les statistiques macro-économiques, les échanges extérieurs ne représentent qu'un cinquième du chiffre d'affaires global de ces petites et moyennes sociétés, mais une PME allemande sur quatre est aujourd'hui ouverte au monde. Ce Mittelstand internationalisé se recrute pour l'essentiel dans les branches industrielles intensives en R\&D sur lesquelles repose la spécialisation sectorielle de l'économie allemande : électrotechnique/mécanique de précision, travail des métaux/construction mécanique et automobile, ainsi que la chimie. S'y ajoutent de manière croissante les services. Or le Mittelstand ne se contente pas de vendre ni d'acheter ses produits à l'étranger -de préférence au sein du marché communautaire -, il y développe aussi une intense activité d'investisseur. Il est vrai que le fort degré d'ouverture de l'économie allemande, la structuration du tissu des entreprises en réseaux de partenariats, de même que la culture entrepreneuriale favorisent un positionnement offensif dans la globalisation, aux effets bénéfiques sur la croissance et l'emploi. Plus que le large éventail d'aides de toutes sortes à l'internationalisation des PME, c'est l'accélération des mutations de l'environnement mondial et européen des activités qui a poussé et pousse toujours les PME allemandes à s'insérer avec succès dans le nouveau partage mondial du travail.
\end{abstract}

\section{Près de 400000 PME actives à l'international}

Constatant que l'économie allemande est de plus en plus largement tributaire de l'évolution de son environnement mondial, et consciente du fait que le Mittelstand constitue ce qu'il est convenu d'appeler outre-Rhin "la colonne vertébrale des activités ", milieux politiques, scientifiques et économiques tentent aujourd'hui de mieux comprendre le rôle des PME dans le nouveau partage mondial du travail. Si cette 'classe moyenne' du tissu des entreprises (c'est le sens même du concept de Mittelstand) a fait l'objet d'une attention particulière depuis les années 1970 et conduit à l'adoption d'une loi fédérale sur le soutien au Mittelstand, c'est que les quelque 3,5 millions de PME qui la composent $(99,7 \%$ du total des sociétés) occupent $70 \%$ des actifs et forment $80 \%$ des apprentis. C'est donc de la prospérité de ces entreprises, majoritairement familiales (voir REA 82/07), que dépendent la santé de l'économie allemande comme la pérennité de son modèle, les PME jouant un rôle actif dans la création du lien social.

Allemagne : structure du tissu d'entreprises (2005)

\begin{tabular}{|cc|}
\hline CA en millions $€$ & Nombre d'entreprises \\
$>1000$ & 385 \\
$500-1000$ & 410 \\
$250-500$ & 950 \\
$50-250$ & 7070 \\
$25-50$ & 8100 \\
$5-25$ & 55860 \\
$1-5$ & 227800 \\
$0,25-1$ & 564600 \\
\hline
\end{tabular}

Source des données : Destatis/KB Deutsche Industriebank, Unternehmerthemen, avril 2007. 
Or paradoxalement, cet ensemble disparate est amplement méconnu des statistiques officielles, et peu d'éléments encore permettent de comprendre son apport réel dans l'ouverture au monde de l'économie allemande. Ce n'est que récemment, depuis l'émergence du débat sur "l'économie de bazar » lancé par le patron de l'institut Ifo de Munich, Hans-Werner Sinn (voir REA 76/06), et entretenu par une vague de délocalisations et de compression d'effectifs dans le groupe des grandes sociétés, qu'on a commencé outre-Rhin à chercher à mieux appréhender l'évolution des stratégies des PME face à la globalisation.

\section{Des statistiques trop globales et des enquêtes très partielles}

Car si les sources de données macro-économiques sur les échanges sont nombreuses (la Bundesbank publie des données sur les investissements, Destatis des statistiques sur le commerce extérieur, etc.), elles ne permettent guère cependant d'avoir une vision précise de la structure par taille des entreprises ouvertes à l'international ou de l'effet des échanges sur l'activité, ni encore moins de comprendre les stratégies du Mittelstand et les motivations qui l'animent dans le choix des partenaires commerciaux.

Destatis publie certes un classement des entreprises par CA ou par appartenance sectorielle, mais ces données sont agrégées. De son côté, l'Institut für Arbeitsmarkt- und Berufsforschung (IAB) qui dépend de l'Agence fédérale pour l'emploi publie, sous l'angle du marché du travail, des statistiques sur les activités internationales des unités de production, classées par taille d'effectif. Selon les dernières données publiées (elles remontent à 2003), les unités de moins de 500 salariés réalisent près de $60 \%$ du CA à l'export de l'économie allemande (et $80 \%$ du CA total). Mais il s'agit là d'unités de production et non d'entreprises, et les données sont agrégées elles aussi.

En un mot : le nombre d'entreprises allemandes varie selon les critères pris en considération, et donc les sources. De plus, il est délicat de faire la part entre les entreprises artisanales (nombre d'entre elles oeuvrent dans l'industrie) et les autres ou, plus important, de prendre en compte le degré réel d'interpénétration entre les diverses activités industrielles et les prestations de services liées. II faut donc compléter ces données agrégées par une série de sondages, dont les enquêtes menées à intervalles réguliers par la fédération de l'Industrie BDI, l'Institut für Mittelstandsforschung et d'autres institutions dont la banque dédiée aux PME, la KfW. Mais ces sondages sont sectoriels, comme ceux de la fédération BDI qui ne s'intéresse qu'à l'industrie, ou le plus souvent partiels: la KfW ou d'autres banques sondent leur clientèle, le corpus de la fédération des CCI allemandes (DIHK) ne tient pas compte des PME artisanales, affiliées à leur chambre de métier (Handwerkskammer)...

Structure des entreprises par taille de l'effectif et par secteur (2003)

\begin{tabular}{|c|c|c|c|c|c|}
\hline & \multirow{2}{*}{$\begin{array}{c}\text { Entreprises } \\
\text { Total }\end{array}$} & \multicolumn{4}{|c|}{... par nombre de salariés } \\
\hline & & $0-9$ & $10-49$ & $50-249$ & $>250$ \\
\hline Extraction de minéraux & 3323 & 2405 & 756 & 128 & 34 \\
\hline Industrie & 289857 & 218633 & 50048 & 16749 & 4427 \\
\hline Eau et énergie & 11847 & 9468 & 1384 & 780 & 215 \\
\hline BTP & 314005 & 279119 & 31628 & 3092 & 166 \\
\hline Commerce (dont réparation automobile et entretien biens de conso.) & 779659 & 700147 & 67113 & 11383 & 1016 \\
\hline Gastronomie/hôtellerie & 264040 & 249213 & 13250 & 1488 & 89 \\
\hline Transports et communications & 146610 & 123576 & 18540 & 3853 & 641 \\
\hline Banque et assurance & 61606 & 52236 & 6046 & 2562 & 762 \\
\hline Immobilier & 851785 & 801159 & 40585 & 8896 & 1145 \\
\hline Education & 69866 & 52998 & 13775 & 2632 & 461 \\
\hline Prestations sociales, Santé (y compris secteur vétérinaire) & 238666 & 205484 & 23272 & 8136 & 1774 \\
\hline Autres services publics et services à la personne & 322574 & 303643 & 15235 & 3165 & 531 \\
\hline Total & 3353838 & 2998081 & 281632 & 62864 & 11261 \\
\hline
\end{tabular}

Source des données : Destatis, Statistisches Jahrbuch 2006. Cet ouvrage contient les dernières données agrégées disponibles.

$98 \%$ des entreprises exportatrices sont des PME selon Destatis
Une institution toutefois a pour mission d'étudier le Mittelstand dans toute sa complexité, d'améliorer sa prise en considération par les statistiques, et de formuler le cas échéant des recommandations aux pouvoirs politiques : l'Institut für Mittelstandsforschung (IfM, Bonn), une fondation de droit privé constituée en 1957 par le Bund et le Land de Rhénanie du Nord-Westphalie. Selon des calculs ad hoc effectués en 2006 pour le compte de cet institut par Destatis sur la base de ses statistiques fiscales de 2004, sur un total de 2957173 entreprises soumises à la taxe sur le chiffre d'affaires (le seuil d'imposition est fixé à un CA supérieur à $17500 €$ depuis 2003), l'Allemagne comptait 342295 entreprises exportatrices, dont 335576 PME. Les 6719 grandes sociétés exportatrices (au CA supérieur à 50 millions $€$ ) totalisent près des quatre cinquièmes du CA à l'export. Les entreprises au CA inférieur, c'est-à-dire les PME selon le seul critère commun aux définitions allemande et communautaire (l'autre critère, portant sur la taille de l'effectif diffère : moins de 500 salariés dans la définition allemande, moins de 250 dans celle de la Commission) ne réalisent que $20 \%$ du total, alors même qu'elles ont une part de $44 \%$ dans le CA domestique. Une petite moitié de ces PME exportatrices était active au sein de l'UE. 
Activités à l'international des entreprises allemandes (2004)

\begin{tabular}{|c|c|c|c|}
\hline & Total entreprises & ... dont PME & Part des PME \\
\hline Nombre d'entreprises & 2957173 & 2948829 & $99,7 \%$ \\
\hline CA total (milliards $€$ ) & 4347,6 & 1730,4 & $39,8 \%$ \\
\hline Entreprises importatrices (biens en provenance de l'UE) & 376498 & 369665 & $98,2 \%$ \\
\hline CA à l'import (biens en provenance de l'UE) en milliards $€$ & 336,0 & 84,1 & $25,0 \%$ \\
\hline Entreprises exportatrices & 342295 & 335576 & $98,0 \%$ \\
\hline CA total des exportatrices (en milliards $€$ ) & 3146,3 & 824,8 & $26,2 \%$ \\
\hline CA à l'export (en milliards €) & 769,9 & 165,1 & $21,4 \%$ \\
\hline - dont export dans l'UE (en milliards €) & 418,8 & 86,1 & $20,6 \%$ \\
\hline - dont export dans pays tiers (en milliards $€)^{*}$ ) & 351,1 & 79,0 & $22,5 \%$ \\
\hline
\end{tabular}

Source des données : IfM, Die Bedeutung der außenwirtschaftlichen Aktivitäten für den Mittelstand, 2007; données établies sur la base de statistiques fiscales (taxe sur le CA) fournies par Destatis en 2006 . Ces données 2004 sont les seules disponibles actuellement. Quant à la définition du groupe des fiscales (taxe sur le CA) fournies par Destatis en 2006. Ces données 2004 sont les seules disponibles actuellement. Quant à la définition du groupe des
$\mathrm{PME}$, elle repose sur le seul critère du chiffre d'affaires (inférieur à 500 millions $€$ ). *) Ces données datant de 2004 , les 12 nouveaux Etats membres $\mathrm{PME}$, elle repose sur le seul critère du chiffre d'affaires (inférieur à 500 millions $€$ ). * ') Ces données datant de 2004 , les 12 nouveaux Etats
intégrés dans l'UE dans le contexte de l'élargissement figurent dans cette rubrique ; l'UE ne comprend donc que les 15 'anciens' membres.

La nature de l'engagement international des PME allemandes

\begin{tabular}{|c|c|c|}
\hline & Nombre & en $\%$ \\
\hline \multicolumn{3}{|l|}{ Commerce extérieur } \\
\hline PME se livrant aux échanges & 364000 & 28,3 \\
\hline - PME exportatrices & 238000 & 18,5 \\
\hline - PME importatrices & 258000 & 20,1 \\
\hline Import seul & 126000 & 9,8 \\
\hline Export seul & 106000 & 8,2 \\
\hline Import et export & 132000 & 10,3 \\
\hline \multicolumn{3}{|l|}{ Investissement direct } \\
\hline PME effectuant des ID & 41000 & 3,1 \\
\hline - sous forme de succursale/filiale & 31000 & 2,4 \\
\hline - sous forme de participation à l'étranger & 21000 & 1,7 \\
\hline - seulement succursale/filiale & 19000 & 1,5 \\
\hline - seulement part de capital & 9000 & 0,7 \\
\hline - les deux & 12000 & 0,9 \\
\hline \multicolumn{3}{|l|}{ Degré d'internationalisation par type d'engagement } \\
\hline PME internationalisées (tous types d'engagement) & 381000 & 29,6 \\
\hline -une seule forme d'engagement & 357000 & 27,8 \\
\hline - import/export seulement & 340000 & 26,4 \\
\hline - ID seulement & 16000 & 1,2 \\
\hline - filiale/succursale seulement & 8000 & 0,6 \\
\hline - part de capital seulement & 4000 & 0,3 \\
\hline - les deux & 4000 & 0,3 \\
\hline - deux formes d'engagement & 24000 & 1,9 \\
\hline - import/export et ID & 24000 & 1,9 \\
\hline - import/export et succursale/filiale & 11000 & 0,8 \\
\hline - import/export et part de capital & 6000 & 0,4 \\
\hline - import/export + succursale/filiale + part de capital & 8000 & 0,6 \\
\hline
\end{tabular}

Source des données : IfM, op.cit.. Calculs effectués par l'IfM sur la base de l'enquête MIND 2005 et des statistiques fiscales (taxe sur le CA) 2003 fournies par Destatis en 2005. NB : Le corpus se compose de PME dans l'industrie et les services au CA compris entre $100000 €$ et 50 millions $€$, de PME du commerce de détail au CA de $250000 €$ à 50 millions $€$ et de PME du commerce de gros au CA de $500000 €$ à 50 millions $€$. Le nombre de PME entrant dans chaque rubrique de ce tableau est calculé sur la base des statistiques 2003 sur le CA établi par taille du CA et par secteur d'activité (base : 1286023 PME).

Dans une précieuse étude sur les activités internationales des PME publiée au printemps 2007, l'IfM a confronté les diverses sources existantes et cherché à en extraire une vision d'ensemble quant à la nature et au degré de l'engagement du Mittelstand. II en appert que 381000 PME pratiquent au moins une forme d'internationalisation : export, et/ou succursale et/ou participation au capital d'une société étrangère. S'y ajoutent 41000 PME réalisant des investissements directs à l'étranger, " soit nettement plus que les 6010 investisseurs recensés par la Bundesbank en 2003 », constate l'IfM. Enfin, quelque 8000 PME recourent à toutes les formes possibles d'ouverture au marché mondial. Autrement dit: les PME allemandes ne se limitent pas au commerce.

A l'évidence, le type d'investissement effectué à l'étranger est fonction de la taille de l'entreprise. Les plus petites préfèrent nettement ouvrir une succursale/filiale hors des frontières; celles au CA compris entre 10 et 50 millions $€$ s'engagent
Les PME allemandes ne sont pas seulement exportatrices

Une stratégie de développement pas à pas hors des frontières 
Les petites PME investissent à l'étranger elles aussi

Trois quarts des ID dans I'UE

Un tiers des PME industrielles internationalisées est doublement engagé à l'étranger

Les PME des services investissent elles aussi parallèlement dans le capital d'une entreprise étrangère. On voit là, traduite en chiffres, la stratégie de développement des PME exportatrices : le premier pas vers la consolidation des activités consiste à ouvrir une succursale ou filiale dans le but d'assurer la fluidité de la production puis des échanges (d'abord les importations, puis les exportations) ; cette 'base' permet la croissance des activités et se prolonge ensuite, le cas échéant, par une prise de participation au capital d'une société sise dans le pays partenaire. Voilà comment les PME allemandes poursuivent une sorte de stratégie de croissance organique hors du territoire national, y 'exportant' pareillement leur mode de fonctionnement en réseau. Elle concerne bien sûr prioritairement les plus performantes et les plus grosses en termes de CA ou d'effectif.

II n'en reste pas moins que la moitié des PME qui investissent (46\% selon les calculs de l'IfM) ont un CA inférieur à 1 million $€$. Ces données, établies sur la base de l'enquête MIND menée annuellement auprès d'un échantillon de 1080 patrons de PME (pour 1,3 million de PME au CA supérieur à $100000 €$ et occupant moins de 500 salariés), peuvent être considérées comme représentatives. Cette enquête, réalisée conjointement par le magazine économique impulse (Gruner+Jahr) et la fédération des caisses d'épargne Deutscher Sparkassen- und Giroverband, bénéficie de l'accompagnement scientifique de l'IfM.

La destination privilégiée de ces investissements est le marché communautaire (UE 15) où s'effectuent les trois quarts des ID (2005). Un petit cinquième des PME ayant ouvert une filiale ou succursale l'a fait dans un des PECO (les nouveaux Etats membres d'aujourd'hui). Une approche par secteurs révèle que ce sont les PME de l'industrie (mais aussi de plus en plus celles des services), qui investissent majoritairement au sein de l'actuelle UE 27 - un état de fait qui correspond à la structuration géographique du commerce extérieur allemand.

Répartition régionale des ID sortants par grands secteurs

\begin{tabular}{|lccr|}
\hline & UE 15 & PECO & a) \\
Industrie & PME ayant une succursale/filiale hors d'Allemagne & \\
bommerce & $71,2 \%$ & $29,9 \%$ & $43,3 \%$ \\
Services & $95,6 \%$ & $36,7 \%$ & $10,3 \%$ \\
Total & $64,5 \%$ & $3,3 \%$ & $35,9 \%$ \\
& $73,6 \%$ & $18,1 \%$ & $31,8 \%$ \\
Industrie & PME ayant une part de capital dans une société étrangère ${ }^{\text {c) }}$ & \\
Commerce & $72,4 \%$ & $18,6 \%$ & $48,3 \%$ \\
Services & $58,2 \%$ & $53,0 \%$ & $7,4 \%$ \\
Total & $83,7 \%$ & $18,1 \%$ & $18,1 \%$ \\
\hline
\end{tabular}

Source des données : IfM, op. cit.. Calculs effectués par l'IfM sur la base de l'enquête Mind 2005. NB. Plusieurs réponses possibles. a) II s'agit des 12 nouveaux Etats membres ayant rejoint l'UE depuis 2004; b) base : 31000 PME disposant d'une succursale/filiale hors d'Allemagne ; b) base : 21000 PME ayant des parts dans le capital d'une société étrangères.

La fédération allemande de l'industrie (Bundesverband der Deutschen Industrie, $B D I)$, de son côté, sonde les entreprises du secteur (critère de définition de la PME : effectif inférieur à 500 salariés) deux fois par an, s'intéressant surtout à leur appréciation de l'évolution conjoncturelle. D'habitude, ces sondages (BDIMittelstandspanel) portent exclusivement sur les exportations (état et prévisions). Or pour la première fois, au printemps 2005, la fédération avait demandé aux sondés d'exposer leur stratégie internationale dans toutes ses formes possibles: de l'investissement à la coopération avec leurs homologues étrangers. II ressort de cette enquête que $68,9 \%$ des PME industrielles ont développé leurs activités à l'international : si 55,6\% se contentent d'exporter, 9,2\% investissent et $26 \%$ ont engagé des liens de coopération. Sur l'ensemble des PME ouvertes au monde, un tiers $(32,5 \%)$ est présent à l'étranger sous deux formes à la fois : export et coopération, export et investissement, ou investissement et coopération. Et 6,6\% des PME pratiquent les trois formes.

Ces résultats coïncident dans une large mesure avec les données obtenues par les enquêtes MIND, révélant l'importance du groupe des PME se livrant aux 
échanges. La seule différence notable entre les deux sources se trouve dans la proportion des entreprises internationalisées qui se contentent d'investir hors des frontières allemandes : elle est de 4,2\% dans l'enquête MIND 2005, et de $2 \%$ seulement dans le sondage du printemps 2005 du BDI. La différence s'explique aisément : le panel du BDI ne comprend que des entreprises industrielles, alors que celui de MIND inclut également le commerce et, par-delà, l'ensemble des services où le choix de l'investissement comme forme d'internationalisation (délocalisation) est de plus en plus souvent déterminé par la facilité et le faible coût des échanges que permettent les applications des TIC.

Le degré d'internationalisation des PME industrielles allemandes est lié à leur taille

\begin{tabular}{|lcccc|}
\hline Type de PME & $\begin{array}{c}\text { PME non } \\
\text { internationalisées }\end{array}$ & $\begin{array}{c}\text {... présentes } \\
\text { dans l'UE }\end{array}$ & $\begin{array}{c}\text { 1.. dans } \\
\mathbf{1} \text { à régions }\end{array}$ & $\begin{array}{c}\text { 4.. dans } \\
\text { Effégions ou plus }\end{array}$ \\
CA moyen (en millions $€$ ) & 24 & 43 & 74 & 372 \\
Marge > 5 \% du CA (en \% des PME) & 4,1 & 7,7 & 13,0 & 57,6 \\
Taux d'exportation (en \%) & 27,8 & 37,5 & 43,7 & 42,1 \\
\hline
\end{tabular}

Source des données : IfM, op. cit. Calculs de l'institut IfM sur la base du BDI-Mittelstandspanel du printemps 2005. Base : 106398 PME industrielles.

De toute évidence, il existe une corrélation entre la taille de l'entreprise (CA, effectif) ou sa rentabilité et son type d'engagement à l'international. Les plus grandes sont nettement plus globalisées que les moyennes qui se contentent du marché communautaire ; quant aux petites, elles ne quittent guère le marché domestique. Visiblement, une certaine taille est requise pour franchir les frontières, tant au plan des ressources humaines que du capital-développement. Or la structuration des PME par taille est aussi largement déterminée par leur appartenance sectorielle : dans les services, les entreprises sont dans l'ensemble plus petites que dans l'industrie, et l'internationalisation se traduit pour elles par moins de coûts fixes que dans la production manufacturière. II faut donc se garder, à partir de cette photographie, d'établir un quelconque lien de causalité entre la puissance des PME et leur degré d'engagement à l'international.

\section{La globalisation a forcé l'ouverture au monde des PME}

II faut ajouter que la structure du tissu des entreprises a considérablement évolué au fil de la décennie écoulée. Ces mutations affectent plus la taille des entreprises que leur nombre, resté relativement stable. Si les structures au CA inférieur à 5 millions $€$ constituent toujours la base du Mittelstand (elles sont actuellement près de 2,3 millions selon les calculs de l'IKB Deutsche Industriebank qui se fondent sur les rares données historiques de Destatis), leur nombre a légèrement baissé depuis les années 1990. Celui des PME moyennes (CA de 5 à 50 millions $€$ ) n'a pas varié. A l'opposé, le groupe des grosses PME s'est nettement accru, augmentant de plus de $20 \%$ dans la catégorie du CA de 50 à 250 millions $€$, voire de plus de $30 \%$ dans la catégorie supérieure (CA inférieur ou égal à 500 millions $€$ ). Cela étant, les statistiques officielles ne permettent guère de croiser ces données avec l'évolution des PME à l'international.

Le 'grossissement' de la classe moyenne des PME allemandes a pour principale origine l'évolution du contexte des activités. L'accélération de la globalisation au cours de la décennie, la rapide diffusion des TIC comme la baisse des coûts liés aux transports et aux communications, l'accélération de l'intégration du marché communautaire, la stabilité monétaire interne créée par la monnaie unique, l'extension rapide du marché communautaire dès avant l'élargissement de l'UE à l'est, de même que l'entrée de la Chine dans l'OMC, ont sensiblement modifié la donne. Un certain nombre de petites entités ont déclaré faillite (Bundesbank, 2006), d'autres ont 'grimpé' dans la classification sous l'effet de l'inflation (leur CA nominal s'était accru alors que la nomenclature statistique n'a pas varié) ou par croissance organique (start ups technologiques). Plus généralement, ce changement semble dû à un mouvement de concentration. Les sondages publiés par la KfW (op. cit.) révèlent par exemple que 7 PME sur 10 ont racheté une autre PME ou procédé à

En dix ans, les PME ont 'grossi'...

... sous l'effet des mutations globales des activités 
Le Mittelstand industriel a réduit l'écart avec les grands groupes

Une concurrence avivée sur le marché domestique

La globalisation a forcé la spécialisation des PME...

... et leur repositionnement dans l'espace européen une fusion au cours de la décennie écoulée. Mais, rappelle la même étude, la croissance externe ne suffit pas à expliquer les changements observés dans la structure du Mittelstand. Certes, c'est dans les secteurs-phares de l'économie allemande, les branches industrielles les plus exportatrices, que la mutation a été la plus nette. Mais à peu de choses près, ces mêmes branches (chimie, métallurgie, construction mécanique, électrotechnique et mécanique de précision) sont aussi les plus importatrices, donc soumises à une concurrence avivée. C'est dans celles-ci que la part des TPE (CA inférieur à 1 million $€$ ) reculait tandis que celle des moyennes entités (CA de 5 à 50 millions $€$ ) croissait. A l'inverse, les créations de micro-sociétés ou TPE se multipliaient dans des secteurs en plein développement (énergie).

La consommation intérieure ayant été morose depuis 1997 (à l'exception des années de la bulle Internet), tout porte à croire que la relative stabilité globale de la structure du Mittelstand est en partie le fruit d'une compétitivité internationale accrue du groupe des PME insérées dans le nouveau partage mondial du travail (industrie et services aux entreprises). Certes, toutes les PME ont vu s'accroître leur rentabilité (+ 44,4\% en termes nominaux de 1997 à 2004 ; Bundesbank, 2006), plus en tout cas que les grands groupes (+11\% seulement), au prix d'une stratégie de consolidation favorisée par ailleurs par une forte modération salariale et des coûts d'investissement et de restructuration réduits en comparaison. On constate en tout cas que les PME industrielles et celles du secteur des services aux entreprises ont enregistré des hausses prononcées de leur rentabilité (respectivement de $+37,5 \%$ et de $+40 \%$ ). En termes de bilan financier comme de rentabilité, elles ont nettement réduit l'écart avec les grands groupes - dans les branches globalisées plus que dans les autres, sans qu'il soit toutefois possible d'étayer ce constat par des statistiques plus détaillées.

Très concrètement, pour les PME allemandes, la globalisation s'est traduite dans un premier temps par le renforcement de la concurrence sur le marché domestique, du fait de l'arrivée en nombre d'acteurs ou de produits étrangers. Elles en ont subi les effets d'abord au niveau des importations : non seulement la nouvelle concurrence étrangère vendait ses produits moins cher que les producteurs nationaux mais, plus important encore, nombre de grands groupes allemands multipliaient dans les pays à bas salaires leurs achats de biens intermédiaires (au début, principalement dans les futurs nouveaux Etats membres) au détriment de leurs sous-traitants allemands. Ensuite, au niveau de la production : nombre de clients stratégiques des PME, souvent de grands groupes, ont transféré leur production hors d'Allemagne, à commencer par l'Europe de l'est. Dans l'enquête MIND 2005, plus de $40 \%$ des PME interrogées estimaient souffrir de cette concurrence globale, comme se plaît à le rappeler une étude publiée en 2006 par la KfW sur la globalisation du Mittelstand.

Mais c'est aussi pour rappeler que, dans le même sondage, elles étaient presque autant $(37 \%)$ à considérer cette situation plutôt comme une chance que comme un risque. Et de fait, nombre de PME allemandes ont saisi l'opportunité de ces mutations qui remettaient en question les positions établies, forçant une nouvelle approche de la situation, d'autant qu'au paroxysme de ces évolutions (2000-03), la demande intérieure était au plus bas et tassait la croissance outre-Rhin. La globalisation, en fragmentant la chaîne de création de valeur au moment même où montaient en puissance les économies émergentes et leur offre de produits de masse low cost, a forcé la spécialisation et la concentration sur un cœur de métier.

Les réponses des PME allemandes ont donc été multiples et, surtout, proactives. Les plus ouvertes ont développé leurs exportations, réduit leur production de biens intermédiaires pour la remplacer par des achats (commandes domestiques et importations) et ont procédé à la délocalisation de leurs sites de production. Cette dernière stratégie était beaucoup moins motivée par un éventuel différentiel salarial que par le fait que les groupes qu'elles fournissaient traditionnellement avaient transféré en nombre leurs sites de production hors d'Allemagne, obligeant ainsi les PME partenaires ou sous-traitantes à les suivre, principalement en 
Europe de l'est. Si cette tendance est le propre de la production manufacturière, elle s'est étendue également aux services, de plus en plus indissociables de l'industrie. Ces stratégies de repositionnement se reflètent dans les motivations poussant les PME à importer et à investir hors d'Allemagne.

Dans quel but les PME allemandes importent biens et services

\begin{tabular}{|lcc|}
\hline & Pour la distribution & Comme biens intermédiaires entrant dans leur propre production \\
Industrie & $35,4 \%$ & $64,6 \%$ \\
Commerce & $89,4 \%$ & $10,6 \%$ \\
Services & $48,3 \%$ & $51,7 \%$ \\
Total & $55,2 \%$ & $44,8 \%$ \\
\hline
\end{tabular}

Source : KfW, Die Globalisierung des Mittelstandes : Chancen und Risiken, février 2006. NB: résultat de deux sondages réalisés en 2004 et 2005 par Creditreform, la KfW et l'institut RWI d'Essen auprès d'un panel de 9000 PME ; plusieurs réponses possibles.

Pourquoi les PME allemandes investissent à l'étranger (motivations classées par importance : 1 = très important ; 5 = sans importance)

\begin{tabular}{|lcccc|}
\hline & Industrie & Commerce & Services & Total \\
Conquérir de nouveaux marchés & 2,1 & 2,0 & 1,8 & 1,9 \\
Préserver les positions sur les marchés existants & 2,0 & 1,6 & 1,7 & 1,8 \\
Sources d'approvisionnement à prix attractifs & 2,7 & 3,4 & 2,9 & 2,9 \\
Différentiel salarial avantageux & 2,9 & 4,3 & 3,3 & 3,3 \\
Salariés qualifiés & 3,3 & 4,1 & 3,2 & 3,4 \\
Environnement de R\&D porteur & 4,5 & 4,4 & 4,1 & 4,3 \\
Subventions & 4,3 & 4,4 & 4,3 & 4,3 \\
\hline
\end{tabular}

Source : KfW, op. cit. NB: résultat de deux sondages réalisés en 2004 et 2005 par Creditreform, la KfW et l'institut RWI d'Essen auprès d'un panel de $9000 \mathrm{PME}$; plusieurs réponses possibles.

Même si le facteur coût a une certaine importance, il est indéniablement secondaire en comparaison des impératifs de croissance et de pérennisation des activités. Selon une autre étude, publiée par l'Institut der Deutschen Wirtschaft proche du BDI (Römer, 2007), un tiers seulement des ID à l'étranger obéit à une logique de compression des coûts, les deux autres tiers étant motivés par la conquête de nouveaux débouchés ou la stabilisation des parts de marché. C'est là qu'il faut voir l'une des principales raisons de la multiplication de succursales/filiales ou d'investissements hors d'Allemagne: elles permettent de comprimer les coûts (transport notamment) par l'achat des biens intermédiaires sur place, d'accroître la clientèle et les marges en produisant et vendant produits et services également sur place et, impératif primordial, d'assurer la pérennité du réseau de partenariats. Autrement dit, via l'engagement à l'étranger, "la mondialisation permet aux entreprises allemandes de projeter à l'étranger leurs... réseaux de sous-traitance, tout en conservant des attaches profondes avec leur base productive nationale ” (Lallement, 2006).

Cette stratégie a certes contribué à créer des emplois à l'étranger, mais elle a plus encore bénéficié à l'emploi domestique. Certes, aucune statistique ne permet de connaître la part précise des emplois détruits, 'délocalisées' ou au contraire créés sur le territoire national. Mais diverses études et enquêtes récentes tentent de remédier à cette lacune. Un premier groupe considère les entreprises sous l'angle de la structure de leur capital, se concentrant particulièrement sur les sociétés familiales qui constituent $95 \%$ du tissu des entreprises outre-Rhin, quelle que soit leur taille. Bien qu'on dénombre dans cette catégorie entre 4000 et 9000 grandes sociétés (CA supérieur à 50 millions $€$, effectif supérieur à 500 salariés), elle coïncide toutefois largement avec le périmètre du Mittelstand. Car ce qui caractérise ce concept spécifique à l'Allemagne, ce sont des critères qualitatifs : indépendance statutaire de la PME, identité entre propriété et responsabilité du patron (voir REA 69/04). Les études sur les sociétés familiales sont donc représentatives de l'évolution du groupe des PME, même si les critères quantitatifs peuvent varier. Ainsi, selon une étude publiée en avril 2007 par l'IfM de Bonn, les entreprises familiales ont créé 400000 emplois dans le monde entre 2003 et 2005, dont la moitié en Allemagne (voir REA 82/07). Selon une autre étude, publiée par le cabinet Deloitte et portant sur les 9645 entreprises au CA supérieur à
En s'internationalisant, les PME mondialisent leurs réseaux

Cette ouverture contribue à la création d'emplois outre-Rhin 
50 millions $€$ recensées dans la base de données Hoppenstedt (elle comprend $80 \%$ des entreprises allemandes, son corpus est plus large que celui des statistiques fiscales de Destatis), si les sociétés à capitaux tendent à privilégier la délocalisation des fonctions intensives en main d'œuvre, les familiales tendent, elles, plutôt à développer hors des frontières nationales les fonctions intensives en savoir. Mais avec un bel ensemble, $60 \%$ de toutes ces entreprises estiment que leur internationalisation a eu des effets positifs sur leurs effectifs domestiques.

L'influence des activités internationales sur l'emploi (par fonctions) dans les entreprises allemandes

\begin{tabular}{|c|c|c|c|c|c|c|}
\hline \multicolumn{4}{|c|}{ Entreprises familiales } & \multicolumn{3}{|c|}{ Autres entreprises } \\
\hline Effectifs... & ... en baisse & ... constants & ... en hausse & ... en baisse & ... constants & ... en hausse \\
\hline Management stratégique & $2,8 \%$ & $41,7 \%$ & $55,5 \%$ & $5,6 \%$ & $44,4 \%$ & $50,0 \%$ \\
\hline Management opérationnel & $8,3 \%$ & $43,1 \%$ & $48,6 \%$ & $11,1 \%$ & $36,1 \%$ & $52,8 \%$ \\
\hline Administration & $8,3 \%$ & $44,5 \%$ & $47,2 \%$ & $11,1 \%$ & $47,2 \%$ & $41,7 \%$ \\
\hline Ingénieurs & $5,6 \%$ & $53,5 \%$ & $40,9 \%$ & $8,6 \%$ & $57,1 \%$ & $34,3 \%$ \\
\hline Techniciens & $12,6 \%$ & $43,7 \%$ & $43,7 \%$ & $14,3 \%$ & $51,4 \%$ & $34,3 \%$ \\
\hline$R \& D$ & $1,4 \%$ & $60,6 \%$ & $38,0 \%$ & $2,8 \%$ & $63,9 \%$ & $33,3 \%$ \\
\hline Production & $29,2 \%$ & $30,5 \%$ & $30,5 \%$ & $31,4 \%$ & $40,0 \%$ & $28,6 \%$ \\
\hline Effectif total en Allemagne & $18,0 \%$ & $16,7 \%$ & $65,3 \%$ & $25,0 \%$ & $16,7 \%$ & $58,3 \%$ \\
\hline
\end{tabular}

Source : Deloitte, Aktive Globalisierung. Chancen für deutsche Familienunternehmer ? mai 2007 (www.deloitte.com/de). Etude réalisée par le Zentrum für Gründungsund Mittelstandsfinanzierung de l'European Business School sur la base d'un sondage effectué auprès de 401 entreprises par l'institut forsa (déc. 2006/janv. 2007).

La globalisation modifie la structure de l'emploi
Un autre groupe d'enquêtes considère les seules sociétés au CA inférieur à 50 millions €. Parmi elles, celle menée en 2006 par l'IfM et publiée par la KfW (Mittelstandsmonitor 2007) révèle que l'effectif global des PME exportatrices a augmenté de 2,6\% entre 2003 et 2005 sur le sol allemand, alors que celui des non exportatrices se réduisait dans le même temps de $3,4 \%$. Cette hausse est encore plus nette $(+8 \%)$ dans le cas des PME ayant une succursale/filiale hors d'Allemagne. Dans le même sondage, $99 \%$ des patrons interrogés affirment que leur engagement hors des frontières non seulement préserve l'emploi sur leur site allemand, mais qu'il se solde également par une hausse générale de l'effectif. Cela étant, les réponses diffèrent entre les PME qui sont déjà internationalisées et celles qui s'apprêtent à franchir le pas : ces dernières estiment à $70 \%$ que leur engagement à venir se traduira par une compression de leurs effectifs en Allemagne. L'écart provient d'une différence d'appréciation, explique la KfW : dans la phase de préparation, les prévisions sont marquées par le court terme et orientées sur les coûts immédiats liés à l'engagement à venir.

Quoi qu'il en soit, et pour en revenir à un niveau macro-économique, la globalisation affecte nécessairement le marché de l'emploi. Ainsi, en Allemagne, on constate une hausse du nombre de salariés travaillant directement ou indirectement à l'export : ils étaient 8,9 millions en 2006, soit $23 \%$ des actifs occupés, contre 5,9 millions seulement en 1995 (15\% du total ; Destatis). Sachant que le Mittelstand occupe $70 \%$ des actifs et que les PME industrielles sont largement ouvertes à l'international, on peut supposer que leur compétitivité a contribué à cette hausse générale, mais sans pouvoir quantifier leur rôle. Ensuite, dans toutes les économies aux salaires élevés, comme l'Allemagne, le partage mondial du travail et ses mutations se traduisent par une évolution de la structure de l'emploi : la baisse de l'emploi industriel est compensée par l'essor du secteur des services ; les secteurs les plus ouverts à la compétition mondiale embauchent de préférence des salariés hautement qualifiés ou spécialisés. C'est l'une des raisons qui explique le haut niveau de chômage parmi les moins qualifiés, mais non la seule. Car l'inadéquation partielle de l'offre du système de formation, de même que certaines rigidités du marché de l'emploi, y contribuent au moins autant, sinon plus, comme le rappellent de nombreuses études (Bundesbank, 2006 ; Klodt, 2007). Pour résumer, les effets sur l'emploi sont indirects : une bonne tenue des exportations et, plus généralement, une insertion heureuse dans le partage mondial du travail, finissent par amener une hausse des investissements sur le marché domestique, celle-ci menant à son tour à une dynamisation de l'emploi. 


\section{Les facteurs culturels de la compétitivité des PME allemandes}

S'il se trouve que les PME internationalisées ont accru et accroissent leurs effectifs, explique la KfW, la raison en réside avant tout dans " un avantage général de productivité " particulier aux entreprises globalisées : insertion dans le partage mondial du travail et compétitivité vont de pair, sans qu'on puisse établir de lien de causalité direct (KfW, 2006). Car pour franchir les frontières, il faut déjà un certain degré de compétitivité ; on constate ainsi que les PME internationalisées sont particulièrement performantes. Le lien entre les deux est bien plus d'ordre culturel qu'économique. L'ouverture, qu'elle soit active (ID à l'étranger, ouverture d'une succursale, export/import) ou passive (actionnaire étranger dans le capital d'une entreprise allemande, concurrence étrangère subie sur le territoire national) s'accompagne en général d'une certaine capacité d'adaptation à la nouvelle donne.

Cette capacité trouve son reflet dans l'effort d'innovation, particulièrement développé parmi les PME ouvertes. On peut y voir le poids de la spécialisation sectorielle de l'économie allemande, puisque ces PME appartiennent en majorité aux secteursclefs de l'industrie, tous situés dans les moyennes et hautes technologies. Mais pardelà, elle repose sur une "aptitude fondamentale pour s'armer contre les risques induits par la globalisation et savoir en saisir les opportunités " (KfW, 2006). Structurellement, la force du made in Germany et des PME allemandes repose sur une grande proximité des entreprises avec la demande du client et dans leur fonctionnement en réseaux de partenariats qui assure la fluidité de la diffusion des savoirs et savoir-faire, y compris en matière d'intelligence économique (voir REA 74/05).

Ces facteurs structurels expliquent pourquoi les entreprises industrielles allemandes sont les plus innovantes d'Europe. Selon Eurostat (enquête CIS4 menée en 2007, voir REA 81/07), le taux d'entreprises industrielles innovantes était de $65 \%$ en Allemagne en 2004 ; elles consacraient aux nouveautés-produit $24 \%$ de leur CA. Mais sans négliger pour autant l'innovation-process : elles sont $37 \%$ dans l'industrie et $36 \%$ dans les services intensifs en savoir. Leurs motivations révèlent l'impératif de compétitivité : $25 \%$ de ces entreprises innovent pour réduire leurs coûts, $27 \%$ pour développer la qualité de leurs produits. L'innovation n'est pas l'apanage des grands groupes. Certes, en volume global, la majeure partie des dépenses d'innovation des entreprises allemandes (quelque 108 milliards $€$ en 2006, selon l'institut ZEW) est imputable aux grands groupes. Mais la part du CA qu'ils consacrent à la R\&D n'est en moyenne que de $5,8 \%$, alors que celle des PME (de 1 à 99 salariés) s'élève à 6,8\% (données 2003), explique une analyse publiée par l'Institut der Deutschen Wirtschaft (Elsenbast, 2007). Et, globalement, la part des PME innovantes s'élève à $71 \%$, poursuit-elle ; chez les petites, ce taux est tout de même de $60 \%$.

L'étude rappelle également quels sont les déclencheurs classiques de l'innovation : la demande du client vient en tête, de même que la stratégie poursuivie par le patron. On voit là l'implication de l'entrepreneur-propriétaire du capital de la PME dans son environnement. La moitié des entreprises est intégrée dans un maillage étroit de partenariats rassemblant clients, sous-traitants, et réseaux de transfert de savoirs. Dans la majorité des cas, et indifféremment de la taille de la $P M E$, la R\&D est financée de préférence sur fonds propres (croissance). Si c'est là un des reflets de l'indépendance des PME allemandes (en termes de capital comme d'affaires), qui a pour revers la crainte d'une prise d'influence par un financier extérieur, elle traduit aussi une attitude plus restrictive des banques. La préférence pour l'auto-financement vaut même lorsque la société bénéficie d'un des nombreux programmes de soutien à l'innovation ; mais dans ce cas, elle est insérée plus étroitement encore dans des réseaux de partenariats $(75 \%$ des entreprises) et plus encline à recourir à un de ses partenaires, notamment un centre de recherche appliquée pour (co-)financer ses activités de R\&D : en effet, la principale caractéristique des programmes de soutien à la $R \& D$ est la constitution ou le développement de réseaux (voir REA 74/05), théoriquement en adéquation avec le schéma national d'innovation allemand.

Partenariat étroit avec le client et auto-financement de la $R \& D$ 
Les préférences des entreprises en matière de financement de la R\&D (1 = très important ; $4=$ sans importance)

\begin{tabular}{|l|cccccc|}
\hline & $\begin{array}{c}\text { Petites } \\
\text { PME }\end{array}$ & $\begin{array}{c}\text { Moyennes } \\
\text { PME }\end{array}$ & $\begin{array}{c}\text { Grosses } \\
\text { PME }\end{array}$ & $\begin{array}{c}\text { Toutes } \\
\text { PME }\end{array}$ & $\begin{array}{c}\text { Entreprises bénéficiant d'un } \\
\text { programme de soutien }\end{array}$ & $\begin{array}{c}\text { Grands } \\
\text { groupes }\end{array}$ \\
Fonds propres & 1,2 & 1,3 & 1,3 & 1,3 & 1,2 & 1,3 \\
Crédit bancaire & 2,3 & 2,6 & 3,0 & 2,4 & 2,5 & 2,8 \\
Partenaires externes & 2,6 & 2,6 & 2,8 & 2,6 & 2,4 & 3,9 \\
Capital-risque & 3,1 & 3,3 & 3,4 & 3,1 & 3,4 \\
\hline
\end{tabular}

Source : IW-Analysen $n^{\circ} 32 / 07$. NB : PME petites : $\mathrm{CA}<1$ million $€$; moyennes : CA de 1 à 50 millions $€ ;$ grosses : CA de 50 à 250 millions $€$.

Les programmes d'aide à l'innovation sont peu adaptés

Peu de R\&D hors de la région d'implantation

Les PME boudent le dispositif de soutien au commerce extérieur
Les PME allemandes montrent un faible intérêt pour les innombrables programmes publics d'aide à l'innovation qui leur sont destinés. La raison : un «problème d'image », comme le formule diplomatiquement une étude sur l'innovation dans le Mittelstand publiée en août 2007 par la fédération allemande des chambres de commerce et d'industrie (DIHK). Les programmes étant très nombreux et de sources multiples (Bund, Länder...), l'offre est perçue comme peu transparente, certes. Mais elle est surtout peu praticable au quotidien pour une petite structure à productivité maximale pour un faible effectif : les procédures administratives étant très lourdes, la charge de travail disproportionnée pour une petite structure en anéantit donc la pertinence, tout particulièrement dans le domaine des hautes technologies. S'y ajoutent l'impossibilité d'engager les travaux de R\&D avant la notification de l'accord, la non rentabilité d'une coopération en dessous d'un certain montant d'aides et, enfin, dans de nombreux cas, le refus des banques de cofinancer le projet. Les seules mesures trouvant la bénédiction des PME sont quelques programmes fédéraux comme PRO INNO II (renforcement de la coopération avec les acteurs de recherche), InnoWatt (accompagnement de la R\&D industrielle dans les nouveaux Länder), INSTI (coaching d'un plan de R\&D), certains programmes des Länder, ou les prêts bonifiés de la KfW. Quant aux programmes européens, outre leur faible lisibilité pour un entrepreneur moyen, de même que leur coût bureaucratique très élevé, les PME allemandes leur reprochent surtout d'être trop orientés sur la recherche fondamentale, donc de négliger ce qui est l'essentiel pour les PME : la demande du marché.

La même étude révèle que les activités de R\&D des PME allemandes sont rarement transfrontalières, « le partenariat régional restant déterminant ». Lorsque l'innovation est menée hors d'Allemagne, c'est essentiellement dans les pays limitrophes comme la France, l'Autriche ou la Pologne, avec des cultures considérées comme familières. Et dans ce cas, l'extension de la R\&D hors du territoire n'est que le prolongement naturel des étapes précédentes d'internationalisation : import/export et investissement. II n'y a pas de contradiction alors avec le fait que, dans l'ensemble, les PME se montrent prudentes quant au transfert à l'étranger de leur $R \& D$, redoutant la perte de leur savoir-faire, le coût des barrières administratives ou les problèmes liés aux différences culturelles. Leurs réticences s'appliquent plutôt aux territoires plus lointains, comme la Chine, dont elles maîtrisent moins la culture et donc les risques liés à un engagement en terre inconnue. En un mot: les PME allemandes cherchent à garder la maîtrise du facteur-clef de leur compétitivité : leur culture réactive et leur capacité d'adaptation à un monde en perpétuel mouvement.

De même, elles boudent les programmes d'incitation à l'internationalisation qui leur sont destinés, constate l'étude Mittelstandsmonitor 2007. Pourtant, ils sont plus de 140, si on additionne ceux des Länder, du Bund, des institutions fédérales, des chambres des métiers, des $\mathrm{CCl}$ (les IHK) ou des chambres de commerce extérieur (Außenhandelskammern, AHK). Ce dispositif offre une large gamme de mesures: elles portent ou bien sur le soutien des activités hors du territoire (participation à des salons professionnels, voyages d'études, études de faisabilité, échanges de salariés, aides à la définition de stratégies, coopérations en matière de recherche) ou bien ont une orientation sectorielle (énergies renouvelables, par exemple) ou bien encore, elles se concentrent sur certaines zones géographiques (PECO, économies émergentes...). 
Part des entreprises ${ }^{\star}$ qui connaissent et, parmi celles-ci, qui recourent aux mesures de soutien au commerce extérieur. Idem pour la connaissance des institutions ad hoc et le recours à leurs services (en \%)

\begin{tabular}{|c|c|c|c|c|c|}
\hline \multicolumn{3}{|c|}{ Dispositif de soutien au commerce extérieur } & \multicolumn{3}{|c|}{ Institutions prestataires } \\
\hline & connaissent & y recourent & & connaissent & y recourent \\
\hline Garanties Hermes & $36 \%$ & $1 \%$ & IHK & $71 \%$ & $11 \%$ \\
\hline Dispositif de conseil au com. ext. & $22 \%$ & $13 \%$ & Banques & $45 \%$ & $8 \%$ \\
\hline Bourses de contact/info & $18 \%$ & $13 \%$ & Ambassades/consulats & $42 \%$ & $8 \%$ \\
\hline Séminaires d'information & $18 \%$ & $41 \%$ & AHK & $27 \%$ & $28 \%$ \\
\hline Infos sur le com. ext. & $17 \%$ & $14 \%$ & Fédérations profes. & $23 \%$ & $7 \%$ \\
\hline Soutien foires/salons étrangers & $9 \%$ & $32 \%$ & Chambres des métiers & $23 \%$ & $13 \%$ \\
\hline Aides à l'export & $7 \%$ & $4 \%$ & bfai ${ }^{b)}$ & $17 \%$ & $15 \%$ \\
\hline Délégations d'affaires & $7 \%$ & $20 \%$ & Centres de soutien à l'éco. & $17 \%$ & $6 \%$ \\
\hline Accompagnement politique & $6 \%$ & $4 \%$ & Ets. publics de soutien à l'éco. ${ }^{c}$ & $16 \%$ & $7 \%$ \\
\hline Pools d'entreprises & $2 \%$ & $56 \%$ & Cabinets conseil & $11 \%$ & $14 \%$ \\
\hline Garanties d'investissement ${ }^{\text {a) }}$ & $2 \%$ & $\approx 0 \%$ & $\mathbf{R K W}^{\mathrm{d})}$ & $2 \%$ & $35 \%$ \\
\hline
\end{tabular}

Source des données: IfM, Mittelstandsmonitor 2007. *) Toutes entreprises, actives à l'international ou non. a) couverture des risques politiques liés à une prise de participation dans une société étrangère ou à l'ouverture du tour de table d'une filiale/succursale à des capitaux étrangers ; b) bfai : Bundesagentur für Außenwirtschaft, centre d'information du ministère fédéral de l'Economie ; c) Bund et Länder ; d) RKW : Rationalisierungs- und Innovationszentrum der Deutschen Wirtschaft.

Ce dispositif n'est guère utilisé que par les grands groupes ; les PME l'ignorent largement (à $53 \%$ ). Et lorsque certaines des mesures sont connues des PME, celles-ci y recourent peu. Ainsi, les garanties Hermes, d'habitude citées en exemple pour illustrer le soutien allemand au commerce extérieur, ne sont connues que d'un tiers des PME, et... $1 \%$ d'entre elles seulement y recourt. II est vrai que ces garanties couvrent les activités dans les pays émergents, et sont donc plutôt pratiquées par les grands groupes. Par contraste, les séminaires d'information connaissent un réel engouement, bien que, eux non plus, ne soient guère connus. Parmi les institutions prestataires de services, les chambres consulaires (IHK), bien qu'ayant une mission statutaire d'information et considérées normalement comme la première adresse pour toute entreprise soucieuse d'élargir son horizon, ne sont guère consultées. Il est vrai que le réseau des AHK est, lui, spécialisé dans la veille économique du pays d'accueil, et relativement fréquenté. En revanche, et ce n'est pas un hasard dans une culture construite sur la subsidiarité et donc l'auto-organisation des acteurs, celles parmi les entreprises, PME en l'occurrence, qui ont connaissance des activités d'accompagnement de l'internationalisation que propose le $\mathrm{RKW}$, y recourent volontiers. Ce réseau d'aide à l'auto-organisation des PME dans le domaine de la compétitivité (rationalisation) et de l'innovation, né en 1921 et recréé en 1950 dans le cadre du Plan Marshall, piloté conjointement par les syndicats, le patronat et des fédérations professionnelles, rassemble aujourd'hui 12 fédérations régionales (Länder) et compte 3700 membres - majoritairement des entreprises.

L'APPROCHE POSITIVE DE LA GLOBALISATION ne concerne pas seulement les quelque $400000 \mathrm{PME}$ internationalisées qu'on peut recenser outre-Rhin. Car leurs activités étant intégrées dans divers réseaux de clients et sous-traitants, leur souci de compétitivité rayonne alentour, tirant les performances de leurs partenaires domestiques. C'est de la sorte que les PME les plus exposées à la concurrence au sein comme hors du pays contribuent à développer aussi la spécialisation des autres qui, peu à peu, se détournent d'une production de masse à laquelle la concurrence des pays à bas salaires a ôté toute justification en Allemagne. Quelques hidden champions, leaders mondiaux dans leur production de niche, poussent leurs partenaires à diversifier eux aussi leur gamme et à se concentrer sur leurs forces: la forte réactivité que leur confèrent les faibles volumes qu'elles produisent. Cet 'effet de taille inversé' leur permet de rester au plus près de la demande évolutive du client, y compris en matière de prestations de services liées aux produits, et ce d'autant plus que leurs circuits décisionnels sont courts. La structure encore largement familiale des PME y concourt, de même que le polycentrisme des activités outre-Rhin qui permet la diversification des business modèles et des contextes réservés aux activités (Bourgeois, 2007).

Mais cette force d'innovation, au sens large, se heurte à un certain nombre de handicaps. Elle repose souvent sur la personnalité du patron; les successions 
(quelque 70000 par an) comportent un risque de perte des savoirs et savoir-faire. Elle repose encore plus largement sur les compétences des salariés ; or les entreprises commencent à manquer de techniciens et d'ingénieurs. Certes, elles forment la majorité des apprentis, mais elles ne décident pas seules des profils de qualification, de moins en mois adéquats, et se heurtent à un illettrisme en expansion (voir REA 66/04 et 69/04). Leur compétitivité exige des moyens. Si leur rentabilité s'est considérablement accrue, que leur taux de fonds propres s'est élevé, que la réforme fiscale du gouvernement Schröder a réduit le poids des prélèvements (Bundesbank, 2006) et que les dispositifs d'aides sont importants, elles se heurtent à toute une série de handicaps. Parmi les principaux qu'elles citent ne figurent pas, contrairement à ce qu'on pourrait croire, un niveau élevé des salaires, au contraire, elles l'estiment en adéquation avec leur haute spécialisation : par contre, ce sont les charges sociales qu'elles considèrent comme trop élevées, et la réglementation du travail, encore trop rigide. Elles s'insurgent également contre un régime fiscal qui favorise les sociétés à capitaux, autrement dit : les grands groupes, au détriment de toutes les autres formes juridiques, de même que contre un régime fiscal des successions qui, malgré de récents aménagements, reste défavorable. Elles s'insurgent enfin contre une paperasse qui atteint des proportions incompatibles avec leurs impératifs de productivité et de compétitivité.

Certes, le gouvernement fédéral a réorienté à l'été 2006 sa généreuse politique de soutien au Mittelstand, mais il s'est contenté d'apporter un peu plus de lisibilité à son dispositif et de reconduire à l'identique le montant des fonds ERP dédié aux PME (4 milliards $€$ en 2007 et 2008). Aucune mesure particulière n'est prévue : indépendamment du fait que toute mesure spécifique «comporterait le risque de favoriser des distorsions de concurrence dans les liens entre PME et grandes sociétés et de grever encore plus les budgets publics ", comme l'explique la Bundesbank (2006), une telle politique serait contraire à la doctrine allemande selon laquelle le rôle des pouvoirs publics doit se limiter autant que faire se peut à créer un cadre favorable à l'activité. Dès lors, s'il convient de lever certains handicaps liés à la structuration même du tissu des PME, les revendications des PME, telles que les formule l'Arbeitsgemeinschaft Mittelstand, un lobby fédérant 8 syndicats professionnels et la moitié du Mittelstand, tiennent en ces quelques mots : «la meilleure politique de soutien au Mittelstand qui soit se résume à une bonne politique économique tout court ». Ce qui, dans leur esprit, se résume à une politique favorable à l'activité.

\section{Indications bibliographiques}

ARBEITSGEMEINSCHAFT MItTELSTAND, Jahresmittelstandsbericht 2007

BOURGEOIS I. (dir.), Allemagne : compétitivité et dynamiques territoriales, Coll. Travaux et Documents du CIRAC, Cergy-Pontoise, juillet 2007

DestaTIS, Konjunkturmotor Export, Materialienband zum Pressegespräch am 30. Mai 2006 in Frankfurt/Main (www.destatis.de)

DEUTSCHE BUNDESBANK, «Zur wirtschaftlichen Situation kleiner und mittlerer Unternehmen in Deutschland seit 1997 », Monatsbericht, décembre 2006, et Kapitalverflechtung mit dem Ausland, Statistische Sonderveröffentlichung 11, juillet 2006

DIHK, Fachkräftebedarf und Image von FuE-Förderprogrammen auf dem Prüfstand. DIHKInnovationsreport 2007, août 2007

ELSENBAST W. et al., Forschungsförderung des deutschen Mittelstandes, Coll. IW-Analysen, n०32/2007

GABEL M., "Financement et vieillissement : le Mittelstand en mutation ", Regards sur l'économie allemande, 69/2004

INSTITUT FÜR MITTELSTANDSFORSCHUNG BONN, Die Bedeutung der außenwirtschaftlichen Aktivitäten für den deutschen Mittelstand. Untersuchung im Auftrag des Bundesministeriums für Wirtschaft und Technologie, Bonn, avril 2007

KFW BANKENGRUPPE (ed.), Mittelstandsmonitor 2007, Francfort, mars 2007, et Die Globalisierung des Mittelstandes : Chancen und Risiken, Francfort, février 2006

Klodt H., Christensen B., "Home Market Effects of Foreign Direct Investment: The Case of Germany », IfW Kiel, Außenwirtschaft, vol. I/2007

LALLEMENT R., "Investissement direct, compétitivité et attractivité ", Regards sur l'économie allemande, $76 / 2006$

RÖMER C., Offshoring - wie viele Jobs gehen ins Ausland? Auslandsinvestitionen, Produktionsverlagerungen und Arbeitsplatzeffekte, Coll. IW-Analysen, n²6/2007. 